\title{
Detection of Cervical Cancer by Irrigation Smear and Cervical Scraping
}

\author{
J. M. MUSKETT,* B.SC. ; A. K. CARTER*; O. G. DODGE, M.D., M.C.PATH.
}

Brit. med. F., 1966, 2, 341-342

Since it was first shown (Papanicolaou and Traut, 1941) that the cytological examination of vaginal secretions could reveal unsuspected gynaecological cancer, various techniques for obtaining suitable cellular preparations have been suggested.

Davis (1962) introduced the irrigation smear, using a disposable plastic pipette (cytopipette) containing a cell-preserving fluid. The fluid is ejected into the upper vagina and irrigates the cervix and fornices. It is then aspirated, carrying with it a sample of the cell population. The pipette containing the cell suspension is then sent to the laboratory for processing. Davis introduced this method, not as a substitute for existing techniques (Ayre scrape and Papanicolaou aspiration smear), but as a method by which women could themselves obtain the specimens. It has been claimed that the irrigation smear is as effective diagnostically as the cervical scrape (Bredahl, Koch, and Stakemann, 1.965).

This laboratory is operating, with the support of the Ministry of Health, a pilot population screening scheme for the detection of cervical cancer (the evolution of this project was described by Smith, Muskett, and Easson in 1965). We started in 1964 a matched series of tests (at the request of the Ministry of Health) to evaluate the comparative merits of the Ayre smear and the Davis cytopipette in the detection of cervical malignancy. We now report the results in a series of 905 women examined by both methods.

\section{Method}

One thousand Davis cytopipettes were issued to clinics operated by the Manchester and Salford City Health Departments, and to four clinics run by the Family Planning Association. Full instructions were sent with each batch of pipettes, and the clinic doctors were asked to take a cytopipette sample, followed by a cervical scrape, on all patients aged 30 to 45 years, preferably on the 10th to 18 th day of the menstrual cycle.

The first 500 pipettes were processed in the laboratory according to Davis's (1962) original method. This proved to be time-consuming - the average time per specimen was eight minutes, including staining and mounting of the slide. The second 500 were processed by the Carbofix method (Davis, 1964), and the average time per specimen was reduced to five minutes.

\section{Results}

The slides were read in batches of at least 30. The Davis slides were all scored first, and later the matching Ayre smears for comparison. The results are shown in Table I. In all the six cases in which the pipette smears showed malignant cells, the Ayre smears revealed them also. In five cases where malignant

TABLE I.-Davis Pipette and Ayre Smear Results in a Series of 905 Well

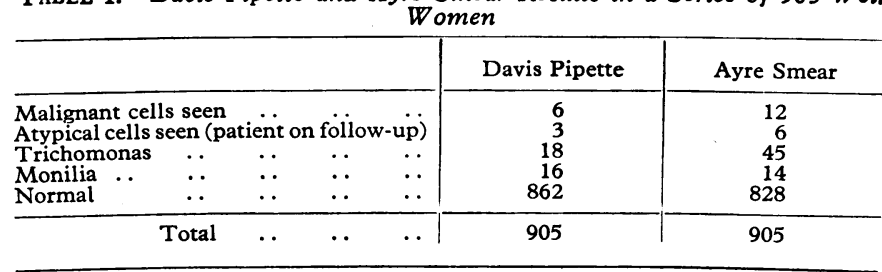

cells were seen in a previous Ayre smear, four were positive in both Davis pipette and Ayre smears, and one was positive in the Ayre smear only. Of the Davis pipettes $6.5 \%$ gave inadequate smears when processed.

Of the 12 cases with positive smears 10 were histologically confirmed as carcinoma-in-situ after cone biopsy, one patient had only a punch biopsy and the diagnosis was not confirmed histologically, and one was reported as chronic cervicitis.

Of the six cases with positive results in the Davis pipette, five were histologically confirmed as carcinoma-in-situ, and one was the patient in whom the diagnosis was not confirmed histologically.

Ayre smears and Davis pipettes were also used, in the same manner, in 30 cases of known invasive cervical carcinoma, with the results shown in Table II.

TABle II.-Davis Pipette and Ayre Smear Results in 30 Cases with Known Cervical Carcinoma

\begin{tabular}{|c|c|c|c|c|c|c|}
\hline & & & & & \multicolumn{2}{|c|}{ Malignant Cells } \\
\hline & & & & & Identified & Not Identified \\
\hline $\begin{array}{l}\text { Davis pipette } \\
\text { Ayre smear }\end{array}$ & 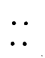 & $\because$ & $\because$ & $\because$ & $\begin{array}{l}19 \\
30\end{array}$ & $\begin{array}{r}11 \\
0\end{array}$ \\
\hline
\end{tabular}

\section{Comment}

\section{Obtaining the Sample in the Clinic}

Various difficulties arose in connexion with the use of the pipettes. Some patients found them unpleasant even though the sample was taken by the medical staff and not on a "do-ityourself " basis. Many women complained of discomfort which was more than transient, and others objected to the staining of their underwear by the residual fluid which was not drawn back into the pipette. The clinic doctors also found the pipettes unpleasant to use, and we found it difficult to complete the series. (It was originally intended to perform 1,000 matched tests.) In addition about $5 \%$ of the pipettes were found to be faulty. The usual fault was splitting of the bulb when this was squeezed to eject the contents of the pipette into the vagina; but also a few pipettes came apart at the junction of stem and bulb. Either of these faults would be distressing to a woman using the pipette on her own.

\section{Processing in the Laboratory}

The processing of the pipettes is tedious and time-consuming compared with the processing of Ayre smears, and sevis ai difficulties were encountered. Of the pipettes $6.5 \%$ gave inadequate smears, either because they contained too little material for adequate evaluation or because of poor cell-preservation. Although it has been claimed that cervical cells are preserved satisfactorily for several days in the Davis fluid, in practice this has not been our experience. Pipettes must be processed within 48 hours of taking the sample. If they are delayed, cellpreservation and nuclear staining rapidly deteriorate, and we have found that the specimen becomes useless. A solution of * Cytology Laboratory, Department of Pathology, Christie Hospital and
Holt Radium Institute, Manchester 20 . 
$40 \%$ alcoholic saline without the coloured indicator gave better fixation.

Some pipettes contain a great deal of mucus, which clots when processed, trapping many of the cells. The technical staff in the laboratory also found the pipettes unpleasant to handle, especially in view of the fact that the stem of the pipette may be contaminated after use.

\section{Reading the Smears}

A Davis pipette smear, at its best, consists of an evenly spread suspension of superficial squamous cells. The complete spectrum of cells seen in the Ayre smear is not present, and endocervical cells are very seldom seen. Endometrial cells, for which a pipette smear is said to be better, are also seen more rarely than in the Ayre smear. Even when the pipettes are processed promptly, we have found that the quality of the cellpreservation and staining is poor by comparison with the Ayre smear. The Davis smears are so homogeneous that they become monotonous to read, and "saturation point" is reached more rapidly. Each slide takes longer to screen than an Ayre smear, since it is more difficult to spot single atypical cells than such cells occurring in groups. With practice and some changes in criteria the screening-time may be reduced, but in no circumstances would we expect it to be less than the time necessary to screen the Ayre smear.

\section{Sensitivity of the Cytopipette}

It will be seen from Table I that the pipette method picks up only half the cases of malignancy found by the Ayre smear. The six Davis pipette smears that were negative when the Ayre smears were positive were re-screened, and in no cases were any abnormal cells found. We believe that the failure to detect malignant cells in these six cases was a sampling error, not a screening error. Macgregor, Fraser, and Mann (1966) have reported a similarly poor detection rate in their recent Aberdeen survey. In those cases where atypical cells indicated the need for close follow-up the pipettes were again only half as accurate as the Ayre smear.
The only advantage of the Davis pipette is that patients can take their own sample without the need to attend a clinic or to see a doctor ; it might therefore be possible to reach a larger proportion of the population at risk. However, the low rate of detection would necessitate repeated screenings of each patient at fairly short time-intervals, and although the initial response might be good, it could prove difficult to persuade women to take repeated samples, especially in view of the complaints from the clinics participating in this survey. Like Macgregor and her colleagues, we think that the cytopipette is not an appropriate instrument for urban-community-screening programmes.

\section{Conclusions}

Our results leave us in no doubt that when a cervical cytology specimen is taken by a doctor the Ayre smear is a more effective method than the Davis cytopipette in the detection of malignancy. The Davis method has been extensively used in Denmark (Bredahl et al., 1965) and in the United States (Davis, 1962). Its greatest advantage is that it can be used as a "do-ityourself " method; it may have a place in screening programmes where facilities for expert gynaecological examination are not widely available. The Ayre scrape is a more efficient method, and its use also ensures that the cervix comes under expert scrutiny. We believe that it is the method of choice for population screening for cervical cancer, in this country at least.

This investigation was carried out at the request of the Ministry of Health. We would like to thank the numerous doctors whose cooperation in taking the specimens made this study possible.

\section{REFERENCES}

Ayre, J. E. (1947). Amer. F. Obstet. Gynec., 53, 609.

Bredahi, E., Koch, F., and Stakemann, G. (1965). Acta cytol. (Philad.), 9, 189

Davis, H. J. (1962). Amer. ₹. Obstet. Gynec., 84, 1017.

- (1964). Personal communication. Method suggested by Dr. J. Kernovsky, Santa Fe Laboratory, Santa Fe, N.M., U.S.A.

Macgregor, J. E., Fraser, M. E., and Mann, E. M. F. (1966). Lancet, 1,252 .

Papanicolaou, G. N., and Traut, H. F. (1941). Amer. F. Obstet. Gynec.,

Smith, J. P., Muskett, J. M., and Easson, E. C. (1965). Lancet, 2, 74.

\section{Preliminary Communications}

\section{Mefenamic Acid and Flufenamic Acid compared with Aspirin and Phenylbutazone in Rheumatoid Arthritis}

Brit. med. F., 1966, 2, 342-343

Aspirin and phenylbutazone (Butazolidin) are well established in the treatment of rheumatoid arthritis. Pharmacological studies have shown both to possess anti-inflammatory properties when measured in the laboratory animal. More recently two anthranilic acid derivatives, mefenamic acid (Ponstan) and flufenamic acid (Arlef), were shown to have the same property (Winder et al., 1962, 1963) and subsequently both have been found useful in patients with rheumatoid arthritis (Coodley, 1963 ; Young, 1963).

As mefenamic acid is now freely available, and as flufenamic acid may become so, it is essential that their relative merits should be compared with existing standard remedies. We report the main findings of a double-blind crossover trial of all four drugs in outpatients with rheumatoid arthritis. The drugs were assessed by a variety of methods, and estimates were made also of the reliability of patients in consuming the prescribed drugs. Details of the trial will be published later in full.

\section{Procedure}

Female outpatients with rheumatoid arthritis (as defined by American Rheumatism Association criteria) of more than one year's duration received, during three consecutive periods of four weeks, mefenamic acid, flufenamic acid, and either aspirin or phenylbutazone in one or other of all possible orders according to a balanced Latin-square design. Both aspirin and phenylbutazone are superior to placebo. Confirmation of this fact seemed unnecessary and ethically undesirable, and these drugs were therefore taken as standards for comparison with the two new drugs. Some patients are sensitive to either aspirin or phenylbutazone, so each drug was included in the design, half the subjects being allotted a sequence containing one drug and half containing the other. Ordinarily the choice depended on the statistical design, but if a patient was believed to react 